Ann. Sci. forest., 1974, 31 (1), 27-45.

\title{
PRINCIPAUX CARACTÈRES \\ ÉCOLOGIQUES ET FLORISTIQUES \\ DES HÊTRAIES \\ DU NORD-EST DE LA FRANCE (1)
}

\author{
J. TIMBAL \\ Laboratoire de Botanique forestière, \\ Centre national de Recherches forestières, I. N. R. A., \\ 14, rue Girardet \\ 54042 Nancy Cedex
}

\begin{abstract}
RÉSUMÉ
Cet article se propose de donner une vue d'ensemble de la typologie des hêtraies que l'on peut trouver aux différents étages de végétation du Nord-Est de la France. Pour chaque type il est donné des indications sur les facteurs écologiques qui le déterminent (substrat-climat) et sur les espèces qui le caractérisent le mieux sur le plan phytosociologique. Un tableau synthétique essaie de faire une récapitulation de l'ensemble et indique la place respective de chacun des types, les uns par rapport aux autres, et par rapport aux facteurs écologiques climat et substrat.
\end{abstract}

\section{INTRODUCTION}

Contrairement à ce qui se passe dans beaucoup de régions de France, le Hêtre est, dans le Nord-Est de notre pays, l'essence la plus répandue. Elle le serait plus encore si en de nombreux endroits, le traitement séculaire en taillis et taillis-sous-futaie, ne l'avait défavorisée et parfois même complètement éliminée au profit des Chênes, du Charme et des autres essences qui rejettent bien de souche après recépage.

Presque partout en Lorraine le Hêtre est l'essence climacique (climax climatique au sens de Duchaufour). Le fait qu'on le trouve sur une grande gamme de sols et sur une large

(1). Communication présentée le 21 novembre 1973 à l'occasion des exposés, sur le Hêtre et le Chêne organisés au C.N.R.F. 
tranche altitudinale le prouve bien. Il n'y a que dans les Vosges où la hêtraie-sapinière remplace la hêtraie comme climax.

Cependant, à basse altitude, dans la plaine lorraine, la hêtraie est éliminée des sols argileux hydromorphes. Ce sont des chênaies-charmaies et des chênaies-frênaies qui la remplacent, formant ainsi ce qu'on appelle, toujours selon Duchaufour, des climax stationnels (ici des climax édaphiques).

Néanmoins, sur cette aire très étendue qui va de la Champagne humide (exclue) à l'Ouest, jusqu'aux sommets des Vosges, à l'Est, et des Ardennes (exclues) au Nord, jusqu'au plateau de Langres au Sud, les peuplements forestiers que forme le Hêtre sont très variés. Cette variété tient tout d'abord à l'altitude, c'est-à-dire, en gros, au climat, à la nature du substrat, et enfin à l'existence de microclimats particuliers liés à la topographie.

Étant donnée l'importance de ces hêtraies dans l'économie forestière de cette région et le peu de renseignements que l'on avait sur leur typologie, il était intéressant et utile d'en faire l'étude phytosociologique.

Cette étude des différents types de hêtraies, nous l'avons donc effectuée durant ces dernières années, principalement dans le cadre du groupe d'étude sur le Hêtre du Centre National des Recherches Forestières. Nous devons remercier ici notre collègue pédologue F. LE TACON pour l'aide efficace qu'il nous a apportée dans la compréhension du déterminisme édaphique de certaines unités phytosociologiques.

Dans cet essai de synthèse, nous nous sommes efforcé, moins de décrire en détail chaque type de hêtraie dans toutes ses composantes écologiques et floristiques, que de bien faire comprendre leur place respective au sein de leur ensemble et de mettre en lumière les facteurs écologiques qui les déterminent et les différencient les uns des autres.

Afin de clarifier notre problème, nous ne parlerons que des groupements climaciques, c'est-à-dire en équilibre avec leur milieu, en laissant volontairement de côté toutes les forêts trop artificialisées du fait de l'intervention plus ou moins heureuse de l'Homme.

De plus, nous allons examiner successivement les différents étages de végétation que l'on rencontre dans le Nord-Est de la France. Ces étages sont de vastes ensembles bioclimatiquement homogènes, du moins à l'échelle régionale, et il leur correspond donc, de grands ensembles phytosociologiques.

\section{1. - LES hÊTRAIES DE L'ÉTAGE COLLINÉEN}

Elles forment, de loin, la plus grande partie des hêtraies du Nord-Est puisque y sont incluses toutes les hêtraies des plateaux calcaires et des collines de Lorraine.

Toute cette région est soumise grosso-modo à un même climat régional que l'on a coutume d'appeler " climat lorrain ».

C'est un climat de transition entre le climat atlantique et le climat continental. C'est un climat rude, aux hivers longs, aux printemps peu marqués et aux étés souvent chauds et caractérisés par l'importance des perturbations orageuses. D'une manière générale les précipitations sont relativement abondantes et à peu près régulièrement réparties tout au long de l'année.

Les données climatiques et météorologiques de Nancy sont caractéristiques de ce climat. 
Les chiffres indiqués ci-dessous sont des moyennes calculées sur de longues périodes (normales).

Températures moyennes annuelles :

maximum : $13,7^{\circ} \mathrm{C}$

moyenne : $9,4{ }_{0} \mathrm{C}$

minimum : $5,1^{\circ} \mathrm{C}$

Pluviosité totale : $726 \mathrm{~mm}$

Nombre de jours de gelée : 80

Nombre de jours de pluie : 70

Nombre de jours de brouillard : 52

Nombre de jours de neige : 20

Nancy est située dans une cuvette à $220 \mathrm{~m}$ d'altitude. Pour les plateaux calcaires qui sont à une altitude plus élevée ( 350 à $420 \mathrm{~m}$ ) la pluviosité totale annuelle est supérieure ou égale à $800 \mathrm{~mm}$ et les températures moyennes inférieures de 1 à $2^{\circ}$ à celles de Nancy.

La rudesse de ce climat (par rapport, par exemple, à celui du Bassin parisien), son caractère continental, donne à cet étage collinéen de Lorraine un caractère submontagnard marqué. Cela y explique l'abondance du Hêtre sur des substrats très variés et son caractère climacique. Si on se dirige vers le Sud, vers des régions à climat plus chaud et moins pluvieux, le Hêtre n'est plus climatique à basse altitude où il est beaucoup plus localisé (climax stationnel) et ne redevient climatique (climax climacique) qu'à des altitudes plus élevées (étage montagnard). Le fait est déjà particulièrement net en Bourgogne.

La grande variété des types de hêtraies que l'on rencontre à l'étage collinéen du NordEst de la France provient donc essentiellement de la variété des conditions édaphiques qui s'y rencontrent et donc, en définitive, de la variété des assises géologiques.

Suivant la nature plus ou moins carbonatée de ce substrat, on a coutume de distinguer les 3 grands types suivants :

- les hêtraies thermo-basophiles dites calcicoles,

- les hêtraies méso-neutrophiles,

- les hêtraies acidiphiles.

Cette distinction en 3 grands types est, bien entendu, schématique. Nos prospections nous ont montré, qu'en fait, il existait tous les types intermédiaires et que, depuis les hêtraies les plus «calcicoles » jusqu'aux hêtraies les plus « acidiphiles », il existait tout un gradient que la réalisation de tableaux phytosociologiques met facilement en évidence.

\section{1. - Les hêtraies « calcicoles》}

On peut les définir comme les hêtraies développées sur une roche-mère calcaire. Mais selon la nature et la teneur en $\mathrm{CaCO}_{3}$ de ce calcaire, peuvent se différencier un certain nombre de types.

En effet, la teneur en $\mathrm{CaCO}_{3}$ conditionne la formation d'une couche d'argile de décarbonatation plus ou moins épaisse. La décarbonatation est plus ou moins superficielle. On peut la mettre facilement en évidence par l'absence d'effervescence au contact de l'acide chlorhydrique par exemple. Plus la teneur en $\mathrm{CaCO}_{3}$ est élevée, moins il y a de formation par 
altération d'argile de décarbonatation, plus le sol sera superficiel et plus la hêtraie aura un caractère thermo-xérophile accusé. Au contraire, plus la teneur en $\mathrm{CaCO}_{3}$ est faible, plus il se formera d'argile de décarbonatation, plus l'épaisseur de sol au-dessus de la roche-mère calcaire sera importante, - du moins en station horizontale — plus les phénomènes pédogénétiques de brunification et de décarbonatation prennent de l'importance et plus le caractère méso-neutrophile tendra à l'emporter sur le caractère thermo-xérophile. La présence de limon en surimposition vient encore renforcer le caractère méso-neutrophile.

La flore, surtout herbacée, étant très sensible à la teneur en $\mathrm{Ca}^{++} \mathrm{du}$ sol, sur les plateaux calcaires de Lorraine, à la série évolutive des sols qui va de la rendzine au sol brun calcique, correspond une gamme de types de hêtraies que l'on peut caractériser parfaitement sur le plan phytosociologique : hêtraies thermo-xérophiles à Cephalanthères sur les sols les plus carbonatés et hêtraies méso-neutrophiles à Asperule odorante sur les sols eutrophes.

Ainsi, dans les calcaires du jurassique supérieur qui forment les Côtes de Meuse, la teneur extrêmement élevée en $\mathrm{CaCO}_{3}$ explique l'abondance, même en station relativement horizontale, de sols peu évolués de type rendzine ou rendzine brunifiée, avec un mull actif moyennement ou très carbonaté. Aussi n'est-il pas étonnant d'y rencontrer des hêtraies qui comptent parmi les plus thermo-xérophiles du Nord-Est de la France.

Les espèces les plus caractéristiques de ces hêtraies calcicoles sèches sont nombreuses. On peut citer le Chêne pubescent (Quercus pubescens $=Q$. lanuginosa), pur ou plus ou moins hybridé avec le Chêne sessile, des graminées comme la Seslérie (Sesleria caerulea) ou le Brachypode penné (Brachypodium pinnatum), des Cypéracées comme certains Carex (Carex alba, C. humilis, C. montana), des Labiées comme la Mélitte (Melittis melissophyllum), ou la Germandrée petit-chêne (Teucrium chamaedrys) et des espèces appartenant à des familles diverses comme le Nerprun purgatif (Rhamnus cathartica), la Phalangère rameuse (Phalangium ramosum $=$ Anthericum ramosum), le Sceau de Salomon odorant (Polygonatum odoratum $=P$. vulgare), le Framboisier des Rochers (Rubus saxatilis), et enfin des Orchidées comme l'incomparable Sabot de Vénus (Cypripedium calceolus), le Céphalanthère palissant (Cephalanthera pallens) ou l'Épipactis rouge (Epipactis atropurpurea).

Phytosociologiquement toutes ces hêtraies thermo-xérophiles sont regroupées dans l'alliance dite des hêtraies à Céphalanthères (Cephalanthero-Fagion).

Sur les Côtes de Moselle, comme par exemple en forêt de Haye, la situation est différente. Une teneur moindre des calcaires bajociens et bathoniens en $\mathrm{CaCO}_{3}$ explique la formation d'une couche d'argile de décarbonatation souvent épaisse, la présence de rendzines brunifiées et, plus souvent encore, de sols bruns calciques. L'humus est généralement de type mull calcique peu carbonaté ou mull eutrophe.

Sur le plan floristique, cela se traduit par une très grande richesse floristique. On y trouve en effet mêlées, une flore thermo-xérophile appauvrie, une flore calcicole banale et une flore méso-neutrophile des sols à mull.

La présence d'un certain nombre d'espèces, et l'absence de certaines autres peuvent permettre de caractériser les stades successifs de la décarbonatation et les différents types de sol qui leur correspondent.

- Ainsi l'absence de Sesleria caerulea, Carex alba et la présence simultanée de Carex montana, Hepatica triloba, Lathyrus vernus, Melica nutans, etc... est indicatrice d'un stade à mull actif peu ou moyennement carbonaté et d'un sol de type rendzine brunifiée. 
- Par contre l'absence des espèces précédemment citées et la présence d'espèces telles que Carex glauca, Carex digitata, Elymus europaeus; Brachypodium silvaticum, etc... indique un stade moins thermo-xérophile (et donc plus méso-neutrophile) à sol brun calcique et mull eutrophe.

Parmi les espèces dites « calcicoles » on peut distinguer les 2 types biologiques principaux qui sont :

- d'une part les espèces herbacées à enracinement surtout superficiel et qui sont souvent plus des thermo-xérophiles que des calcicoles,

- et, d'autre part, les espèces ligneuses qui composent la strate arbustive, qui possèdent un système racinaire plus puissant et plus profond, capable d'atteindre la roche-mère calcaire au-delà de la couche d'argile de décarbonatation et qui sont souvent plus «calcicoles » que thermo-xérophiles.

De plus, parmi les espèces qui rentrent dans ces deux groupes, toutes ne sont pas calcicoles ou thermo-xérophiles au même degré. Cela dépend en grande partie de leur amplitude écologique vis à vis du pH (voir annexe).

Cela explique que, quand l'épaisseur de la couche d'argile de décarbonatation augmente et qu'on passe d'un sol peu évolué et superficiel à un sol plus évolué dans le sens de la décarbonatation, on peut lire très fidèlement cette évolution dans la flore du sous-bois.

Il est ainsi logique que ce soient les thermo-xérophytes herbacées (la Seslérie par exemple) qui disparaissent les premières quand l'épaisseur de la couche d'argile de décarbonatation augmente; en même temps qu'apparaissent des espèces à caractère méso-neutrophile de plus en plus accentué.

Au stade sol brun calcique, les espèces herbacées sont déjà presque toutes des mésoneutrophiles tandis qu'il existe encore des calcicoles ligneuses telles que les Cornouillers mâle et sanguin (Cornus mas et C. sanguinea), ou les Aubépines (Crataegus oxyacantha et C. monogyna).

On peut dire que l'on sera arrivé à une hêtraie méso-neutrophile typique quand ces dernières calcicoles auront dipsaru à leur tour et qu'il n'y aura plus que des espèces mésoneutrophiles.

Après ce que nous venons d'affirmer, on peut se demander à juste titre où, et à quel stade, doit passer la limite entre hêtraies thermo-xérophiles à Cephalanthère et hêtraies mésoneutrophiles à Aspérule odorante. Les phytosociologues ne sont pas encore d'accord à ce sujet et leur opinion varie suivant la conception plus ou moins large qu'ils se font de ces deux différents groupes. Mais peu importe en pratique. L'essentiel est de bien comprendre le sens du gradient et la variation édaphique qui le détermine.

Pour le forestier il suffit de saisir que ce gradient correspond sur le plan sylvicole à des possibilités de croissance et de régénération naturelle toujours meilleures quand on passe des hêtraies à Cephalanthères sur rendzines aux hêtraies à Asperule sur sol brun. Les hêtraies à Cephalanthères sont en effet très souvent difficiles à régénérer naturellement du fait de l'extrême faiblesse de leur réserve en eau et de la concurrence des « morts bois » calcicoles. De plus, dans certains peuplements très ouverts la Seslérie peut former des tapis continus faisant obstacle à toute germination. Nous retrouverons des problèmes semblables dans les hêtraies acidiphiles. 


\section{2. - Les hêtraies méso-neutrophiles}

Par définition, les hêtraies méso-neutrophiles sont donc celles où l'on ne rencontre que des espèces méso-neutrophiles.

Ces espèces sont nombreuses. Parmi les plus caractéristiques et les plus communes on peut citer des graminées comme la Mélique (Melica uniflora), le Millet (Milium effusum), la Canche caespiteuse (Deschampsia caespitosa), des Cypéracées comme la Laiche des Bois (Carex silvatica) ou la Laiche à nombreuses racines (Carex polyrhyza), et d'autres espèces comme 1'Aspérule odorante (Asperula odorata), le Lamier jaune (Lamium galeobdolon), etc...

Le sol de cette hêtraie est typiquement un sol brun eutrophe et l'humus un mull eutrophe.

La richesse chimique et les réserves en eau du sol sont assez élevées pour que le Hêtre y trouve des conditions optimum pour sa croissance et s'y régénère naturellement avec d'autant plus de facilités que les espèces ligneuses arbustives sont peu abondantes. Seule la Ronce peut, dans certains cas, poser des problèmes sérieux.

Cette hêtraie, du moins dans sa forme typique, est très rare sur les plateaux calcaires de Lorraine tels que le plateau de Haye, où les espèces ligneuses calcicoles sont presque toujours présentes. Cette flore ligneuse calcicole disparaît cependant pratiquement complètement quand sur les argiles de décarbonatation est parfois venue se surimposer une couche plus ou moins épaisse de Limon ou d'alluvions anciennes de la Moselle.

Mais dans ce cas, sur ces derniers substrats, se produit un autre phénomène pédogénétique : le lessivage, qui provoque le passage d'un type méso-neutrophile à un type acidiphile de hêtraie et une légère baisse du niveau de festilité du sol.

Ce passage vers un type acidiphile se traduit dans la flore par certaines modifications aisément perceptibles. Il y a apparition au sein des espèces méso-neutrophiles de certaines espèces à tendance neutroacidiphiles ou acidiphiles. Ainsi Milium effusum tend à remplacer complètement Melica uniflora, et il y a apparition d'espèces du mull mésotrophe ou du mull acide comme Oxalis acetosella, Lonicera periclymenum, Luzula albida, etc...

D'un point de vue phytosociologique, il convient cependant d'inclure dans les hêtraies méso-neutrophiles (hêtraies dites à Aspérule), à la fois l'aile basocline des hêtraies, disons neutro-basophiles, eutrophes, se développant sur argile de décarbonatation épaisse (hêtraies à Carex digitata) et l'aile acidocline des hêtraies, disons neutro-acidiphiles, mésotrophes, se développant sur les placages de limons et d'alluvions anciennes (hêtraies à Milium effusum).

\section{3. - Les hêtraies acidiphiles}

Ce sont les hêtraies développées sur substrat « acide». Cette « acidité » peut être «primaire », c'est-à-dire liée à la pauvreté chimique et principalement en $\mathrm{CaCO}^{3}$ de la rochemère (grès des Vosges du Trias moyen ou grès rhétien du Trias supérieur); ou elle peut être «secondaire », c'est-à-dire consécutive à un phénomène pédogénétique tel que le lessivage ou la podzolisation. Le lessivage est en particulier de règle sur les limons, ce qui explique qu'au sein des chênaies-charmaies qui couvrent les argiles et les marnes du Keuper par exemple, on trouve un îlot de hêtraie acidiphile chaque fois qu'il y a un placage de limons.

Ici aussi la variabilité édaphique entraîne une variabilité parallèle des types de hêtraies 
acidiphiles. II n'y a pas un type unique de hêtraie acidiphile mais toute une série en fonction du degré d'acidité du substrat, c'est-à-dire du taux de saturation du sol et en fin de compte, de la richesse en éléments minéraux de la roche-mère. Il n'y a aucune commune mesure, en particulier sur le plan des possibilités forestières, entre par exemple, une hêtraie installée sur un sol brun acide, profond, développé sur un colluvium de pente ou sur grès intermédiaire et une hêtraie à sol podzolique que l'on peut trouver sur les grès vosgiens.

Comme pour les hêtraies « calcicoles », l'examen de la flore permet de suivre avec précision cette variation pédologique. La flore, et surtout la flore herbacée, est très sensible à la teneur en éléments minéraux, et surtout en calcium, du sol. Aussi reflète-t-elle dans sa composition toutes ces variations édaphiques.

Ainsi la présence simultanée d'espèces neutrophiles comme Melica uniflora, Milium effusum, Carex polyrhyza, d'espèces neutro-acidiphiles comme Oxalis acetosella, Lonicera periclymenum, etc., et d'espèces acidiphiles comme Luzula albida, Polytrichum formosum, Carex pilulifera, Pteridium aquilinum, etc... est toujours indicatrice d'un humus de type mull oligotrophe ou même mull mésotrophe et d'un sol de type brun acide toujours très favorable à la croissance de résineux tels que le Sapin ou le Douglas. Ce type de hêtraie représente ce que nous appellerons l'aile neutro-basocline des hêtraies acidiphiles.

Même quand dans le tapis herbacé, il n'y a que des espèces acidiphiles, on peut encore suivre les variations édaphiques, car toutes les espèces ne sont pas acidiphiles au même degré. Ainsi pour les espèces suivantes on peut établir un classement qui corresponde à un gradient d'acidité croissante :

\section{Oxalis acetosella \\ Luzula albida \\ Deschampsia flexuosa \\ Vaccinium myrtillus \\ Leucobryum glaucum \\ Calluna vulgaris}

Ainsi les hêtraies à Luzula albida dominant sont elles généralement des hêtraies à mull acide. Celles à Deschampsia flexuosa dominant des hêtraies à mull-moder. Celles àVaccinium myrtillus des hêtraies à moder et enfin celles à Leucobryum glaucum et Calluna vulgaris correspondent généralement à des mor-moder ou même, rarement il est vrai, à des mor. Le caractère indicateur de ces espèces acidiphiles est cependant assez relatif dans la mesure où des Ardennes belges à la plaine de la Saône on peut observer un gradient climatique qui semble correspondre à l'importance relative des influences atlantiques et continentales. Par le jeu toujours complexe des compensations de facteurs écologiques et de leur influence sur la pédogénèse, à ce même groupe d'espèces acidiphiles correspondent des humus et des types de sol d'autant plus évolué que les influences atlantiques sont plus fortes. Ainsi d'après P. Roisin, Luzula albida correspond à des moder en Ardennes occidentales et à des mullmoder plus vers le Sud-Est. De même, d'après nos observations personnelles; Luzula albida (mais aussi Deschampsia flexuosa et Vaccinium myrtillus, toujours avec le classement que nous avons indiqué) correspondent à des sols et à des humus de moins en moins évolués quand venant de la région d'Épinal on se dirige vers le Bassin de la Haute-Saône par Darney et Luxeuil. 
Il va sans dire que chaque fois qu'on descend d'une marche dans ce gradient d'acidité, le niveau de fertilité générale diminue, ainsi que les possibilités sylvicoles.

Les hêtraies à Leucobryum glaucum et Calluna vulgaris représentent le type le plus acidiphile que l'on puisse rencontrer dans le Nord-Est de la France. Au-delà de cette échelle, sur des sols podzoliques ou des podzols, et avec des humus tournant franchement au mor, le Hêtre ne peut plus pousser et il est remplacé par le Chêne séssile.

Outre les neutrophiles, les neutro-acidiphiles et les acidiphiles, un $4^{\mathrm{e}}$ groupe d'espèces peut se rencontrer dans les hêtraies acidiphiles de l'étage collinéen. Il s'agit des espèces hygrophiles comme la Molinie (Molinia caerulea) et la Bourdaine (Rhamnus frangula).

Sur substrats limoneux ou alluvial, ces espèces hygrophiles sont généralement indicatrices de l'existence d'une nappe perchée et donc de phénomènes d'hydromorphie avec tout ce que cela comporte comme conséquences dans la gestion forestière (danger de remontée brutale de la nappe par coupe excessive, entraînant un développement « explosif » des espèces sociales héliophiles telles que la Molinie, et l'impossibilité de la régénération naturelle).

Pour terminer ce paragraphe consacré aux hêtraies acidiphiles, il est nécessaire de dire quelques mots de l'Argonne.

Dans cette région bien indivıdualisée géographiquement, on trouve à la faveur d'affleurements acides de Gaize, des hêtraies acidiphiles assez semblables au premier abord à celles de Lorraine. Cependant la présence en leur sein de certains éléments floristiques, leur confère une originalité et un caractère de transition marqué. Ces hêtraies acidiphiles d'Argonne sont donc intermédiaires entre les hêtraies à Luzula albida, très médio-européennes de Lorraine (Luzulo-Fagion), les hêtraies-chênaies acidiphiles atlantiques du Bassin parisien (Quercion r.p.) et les hêtraies acidiphiles montagnardes des Ardennes. Ainsi la présence du Néflier (Mespilus germanica) et la rareté de la Luzule blanchâtre (Luzula albida) sont des traits qui rapprochent les hêtraies acidiphiles de l'Argonne de leurs homologues du Bassin parisien ou de l'Ouest de la France. Par contre, la présence, parfois en abondance, de Calamagrostis arundinacea, espèce absente des hêtraies lorraines, est un élément de ressemblance avec les hêtraies ardennaises.

Ces caractères phytogéographiques particuliers expliquent la place spéciale que nous avons réservée aux hêtraies acidiphiles de l'Argonne dans notre tableau récapitulatif.

\section{4. - Les hêtraies des versants froids}

Il existe enfin un autre type de hêtraie à l'étage collinéen. Ce sont les hêtraies riches en Érable (Acer pseudo-platanus et A. platanoides) et Orme de montagne (Ulmus scabra) que l'on rencontre sur certains versants des ravins qui entaillent les plateaux calcaires ou sur les rebords nord de ces mêmes plateaux.

Leur déterminisme n'est pas édaphique mais microclimatique cette fois. En effet, leur position topographique fait, qu'à leur contact, il y a accumulation de masses d'air froid. En fait ce sont de véritables hêtraies montagnardes qui sont ainsi enclavées dans le collinéen.

Le substrat étant calcaire, on y trouve un certain nombre d'espèces des hêtraies calcicoles comme Carex digitata, Asarum europaeum, Ribes alpinum, etc. Mercurialis perennis y est aussi parfois particulièrement abondant. Mais la présence d'espèces, rares en plaine et 
communes à l'étage montagnard, comme Dentaria pinnata, et Actaea spicata, témoigne de ce microclimat particulier.

Le phytogéographe Morand a récemment montré dans le Laonnois, que, sur une tourbière adossée à un tel groupement, même en plein été, il gelait très fréquemment. $\mathrm{Ne}$ doutons pas que des mesures microclimatiques précises et étalées sur une période assez longue, démontreront avec clarté, qu'il n'est pas besoin de faire appel à la notion de station relique pour expliquer la présence en plaine d'espèces aussi typiquement montagnardes.

\section{2. - LES hÊTRAiES MONTAGNARDES DE LA CHAINE VOSGIENNE}

Si certaines espèces fréquentes en montagne se retrouvent, avec une abondance moindre il est vrai, en plaine (nous les appellerons pour cela submontagnardes), d'autres restent plus ou moins strictement limitées à la zone montagneuse et leurs limites altitudinales inférieures constituent de bonnes limites bioclimatiques.

Il en est ainsi pour des essences comme le Sapin (Abies alba), l'Épicéa (Picea abies), mais aussi pour des espèces herbacées comme le Prenanthe (Prenanthes purpurea), la Grande Fétuque (Festuca silvatica), le Sceau de Salomon verticillé (Polygonatum verticillatum), etc...

Ces limites altitudinales d'aires, nous les utiliserons pour définir avec précision les trois sous-étages inférieur, moyen et supérieur, en lesquels on subdivise ordinairement l'étage montagnard.

A chacune de ces subdivisions correspond donc un contexte bioclimatique différent. Pour ce qui est du climat, cela correspond, du montagnard inférieur au montagnard supérieur, à une augmentation de la pluviosité moyenne annuelle à une diminution de la température moyenne annuelle et à une diminution de la durée de la saison de végétation.

Un autre trait essentiel va caractériser cet étage montagnard des Vosges. C'est l'absence totale de roches carbonatées. Il en résulte que tous les groupements végétaux, à quelque altitude que ce soit, se situent dans la série acidiphile. Cela explique que la variété des types de hêtraies y soit beaucoup moins grande qu'à l'étage collinéen.

\section{1. - Étage montagnard inférieur}

Par définition, nous faisons débuter l'étage montagnard inférieur à la limite altitudinale inférieure du Prénanthe pourpré (Prenanthes purpurea). Sur le versant occidental des Vosges qui nous intéresse cela correspond à une altitude d'environ $350 \mathrm{~m}$. Ce chiffre n'a évidemment rien d'absolu et cette altitude peut varier en fonction du sol et de l'exposition. De plus cette limite est souvent masquée par le fait, qu'à la limite de ses possibilités, le Prénanthe, à cette altitude, est éliminé des sols trop acides (humus de types mor et mor-moder).

Deux types principaux de hêtraies se rencontrent à cet étage montagnard inférieur des Vosges. Le principal est le type à Luzule blanchâtre et Prenanthe pourpré, l'autre le type à grande Fétuque. Tous deux se classent cependant sur le plan phytosociologique dans le grand groupe des hêtraies à Luzule blanchâtre (alliance du Luzulo-Fagion). 


\subsection{La hêtraie à Luzule blanchâtre et Prenanthe.}

La présence du Prenanthe et d'autres espèces montagnardes comme le Gaillet des rochers (Galium saxatile) permet de différencier sur le plan floristique cette hêtraie à Luzule blanchâtre (Luzula albida) de son homologue du collinéen.

Nous retrouvons dans ce type de hêtraie toutes les variances édaphiques que nous avons déjà citées pour les hêtraies acidiphiles de l'étage collinéen. Nous n'en dirons donc pas plus, sinon que développée le plus souvent sur des grès d'une très grande pauvreté chimique, on ne trouve que très rarement la variante neutro-acidiphile à Mélique ou Milium qui est toujours localisée sur les colluvions de bas de pente.

\subsection{La hêtraie à Prenanthe et grande Fétuque.}

Ce type de hêtraie est rare dans les Vosges. Il s'y situe d'abord dans une tranche altitudinale légèrement supérieure au type précédent, disons à la partie supérieure de l'étage montagnard inférieur, vers $400 \mathrm{~m}$ d'altitude.

Il est aussi géographiquement très localisé. On ne le trouve que dans cette région du Col de Saverne, là où, du moins dans les Vosges, le Sapin atteignant sa limite Nord, la sapinière fait place à la hêtraie.

De plus, sur le plan édaphique, les exigences de la grande Fétuque (Festuca silvatica) font que ce type de hêtraie indique toujours des sols bruns acides, relativement profonds et relativement riches en éléments minéraux.

Outre la grande Fétuque, ce type de hêtraie se caractérise floristiquement par l'abondance d'espèces comme le Sureau rouge (Sambucus racemosa), la Moehringie (Moehringia trinervia), la Fétuque géante (Festuca gigantea), etc...

La fertilité de ce type de hêtraie est très grande. Nous y avons mesuré pour le Hêtre des hauteurs dominantes de plus de $40 \mathrm{~m}$. Cependant, sur le plan sylvicole, ces hêtraies à grande Fétuque posent des problèmes au moment de leur mise en régénération, car une ouverture brutale du couvert entraîne un formidable développement de la grande Fétuque qui recouvre entièrement le sol aux dépens des germinations de Hêtre.

Si ce type de hêtraie est si localisé géographiquement, c'est que l'on constate que la limite altitudinale inférieure de la grande Fétuque devient de plus en plus élevée au fur et à mesure que l'on va du Nord au Sud.

Dans les Ardennes la hêtraie à Festuca silvatica existe à basse altitude sur des versants ombragés d'exposition fraîche. Prenanthes purpurea n'y existe pas ce qui montre que ces hêtraies sont à rattacher à l'étage collinéen, mais un collinéen submontagnard un peu comme les hêtraies à Dentaria pinnata des versants froids des plateaux calcaires de Lorraine.

Dans les Vosges du Nord Festuca silvatica entre donc dans la composition d'une hêtraie à Prenanthes purpurea du montagnard inférieur.

Par contre, dans les Vosges Centrales (région de Saint-Dié, Gérardmer), Festuca silvatica devient une espèce de la hêtraie-sapinière (étage montagnard moyen), et on a des sapinières à Fétuque.

Plus au Sud encore, dans le territoire de Belfort, sur le versant méridional des Vosges, Festuca silvatica est une espèce de la hêtraie sommitale vers $800 \mathrm{~m}$ d'altitude (étage montagnard supérieur à subalpin). 
Comme pour les espèces acidiphiles vues précédemment, il est vraisemblable que cette variation écologique est liée à la septentrionalité et à l'importance relative des influences atlantiques et continentales.

\section{2. - L'étage montagnard moyen}

L'étage montagnard moyen débute par définition à la limite altitudinale inférieure du Sapin.

Il n'y a normalement pas de hêtraie à cet étage. La formation climacique est la hêtraiesapinière que l'Homme a le plus souvent transformée pour des raisons économiques et sylvicoles en sapinière pure.

Cependant, sur certains substrats particulièrement « riches » comme les grès intermédiaires, le Hêtre peut devenir envahissant, et par le jeu d'une régénération en Sapin manquée, on peut trouver de place en place des îlots de hêtraie à peu près pure. Mais il s'agit alors plus d'un faciès à Hêtre de la hêtraie-sapinière, que d'une véritable hêtraie du montagnard moyen.

Sur le sol très acide ces îlots de hêtraie ne diffèrent guère sur le plan floristique des hêtraies acidiphiles du montagnard inférieur à Luzula albida, Prenanthes purpurea, Galium saxatile. Par contre un sol plus riche permet l'apparition d'espèces nouvelles comme la Stellaire des Bois (Stellaria nemorum), le Compagnon rouge (Melandrium rubrum), l'Impatiente (Impatiens Noli-tangere), la grande Fétuque (Festuca silvatica), etc...

\section{4. - L'étage montagnard supérieur}

Sa limite inférieure est beaucoup moins nette que celle de l'étage montagnard moyen. C'est l'étage de la hêtraie-sapinière mêlée d'Épicéa.

Ici aussi, normalement, on ne trouve pas de hêtraie. Nous n'en dirons donc pas plus.

\section{3. - L'ÉTAGE « SUBALPIN » DES VOSGES}

Dans les Vosges occidentales, vers $900 \mathrm{~m}$, la hêtraie-sapinière mêlée d'Épicéa disparaît pour faire place à une hêtraie pure, et cela jusqu'à la limite supérieure de la végétation forestière.

Les phytogéographes discutent encore beaucoup, pour savoir si cette hêtraie sommitale des Vosges devrait être encore rattachée au montagnard supérieur, ou constituait une sorte d'étage subalpin. C'est surtout le phytosociologue alsacien R. CARBIENER qui soutient cette dernière thèse en l'appuyant sur de solides arguments phytosociologiques et pédologiques.

Le climat qui règne au niveau de cette hêtraie est particulièrement rude. Le vent et la neige y jouent un rôle très important. La durée de la saison de végétation est relativement courte. Les sols portent l'empreinte du climat. Ce sont des rankers de type pseudo-alpin.

Sur le plan floristique on y trouve un certain nombre d'espèces typiquement montagnardes et même subalpines telles que la Laitue des Alpes (Cicerbita alpina), l'Oseille à feuilles d'Arum (Rumex arifolius), l'Adenostyle à feuilles d'Alliaire (Adenostyles alliariae), la 
Renouée Bistorte (Polygonum bistorta), le Camerisier noir (Lonicera nigra), le Sceau de Salomon verticillé (Polygonatum verticillatum), et un certain nombre de fougères telles que Dryopteris linnaeana et Dryopteris phegopteris.

La variabilité édaphique due à la plus ou moins grande richesse chimique du substrat cristallin n'a que peu de répercussions sur la flore. A cette altitude, ou plutôt à cet étage bioclimatique, l'influence des facteurs climatiques est absolument prépondérante par rapport aux facteurs édaphiques. Sur le plan phytosociologique on peut considérer que toute cette hêtraie subalpine relève d'un seul type phytosociologique et que les variations édaphiques n'induisent que des faciès de ce groupement :

- faciès eutrophe à Rumex arifolius,

- faciès mésotrophe à Milium effusum et Asperula odorata,

- faciès oligotrophe à Deschampsia flexuosa et Vaccinium myrtillus.

On peut cependant distinguer la base de cette hêtraie, celle qui est au contact de la hêtraiesapinière. On y trouve encore du Sapin et de l'Épicéa dans la strate arborescente et dans la strate herbacée seule Polygonatum verticillatum indique le passage au subalpin.

\section{CONCLUSION}

Ce rapide «panorama » des types de hêtraies du Nord-Est de la France nous a permis de nous rendre compte de leur importance, de leur répartition, de leur déterminisme et de leur variété.

Pour le déterminisme, nous avons pu remarquer l'importance respective des facteurs édaphiques et climatiques. Prépondérante à basse altitude la variabilité édaphique y provoque la formation d'un grand nombre de types de hêtraies aux caractéristiques écologiques et aux possibilités sylvicoles différentes. Mais plus on va en altitude plus c'est le facteur climatique qui devient limitant et la hêtraie sommitale des Vosges en est un exemple parfait.

Un autre aspect peut retenir l'attention après ce bref tour d'horizon : c'est le contraste entre le collinéen de la plaine et des plateaux lorrains et le montagnard des Vosges. A l'étage collinéen les hêtraies occupent une énorme surface, c'est la formation climacique, et parmi elles les hêtraies «calcicoles" sont, de loin, les plus importantes. A l'étage montagnard des Vosges, la hêtraie ne joue qu'un rôle mineur par rapport à la hêtraie-sapinière et les formes calcicoles en sont totalement absentes.

Le tableau 1 tente de résumer ces diverses constatations. Nous y avons porté, en abscisse, la variation édaphique et, en ordonnée, la variation bioclimatique. A chacune des cases correspond donc un type principal de hêtraies avec les espèces qui les caractérisent le mieux. 


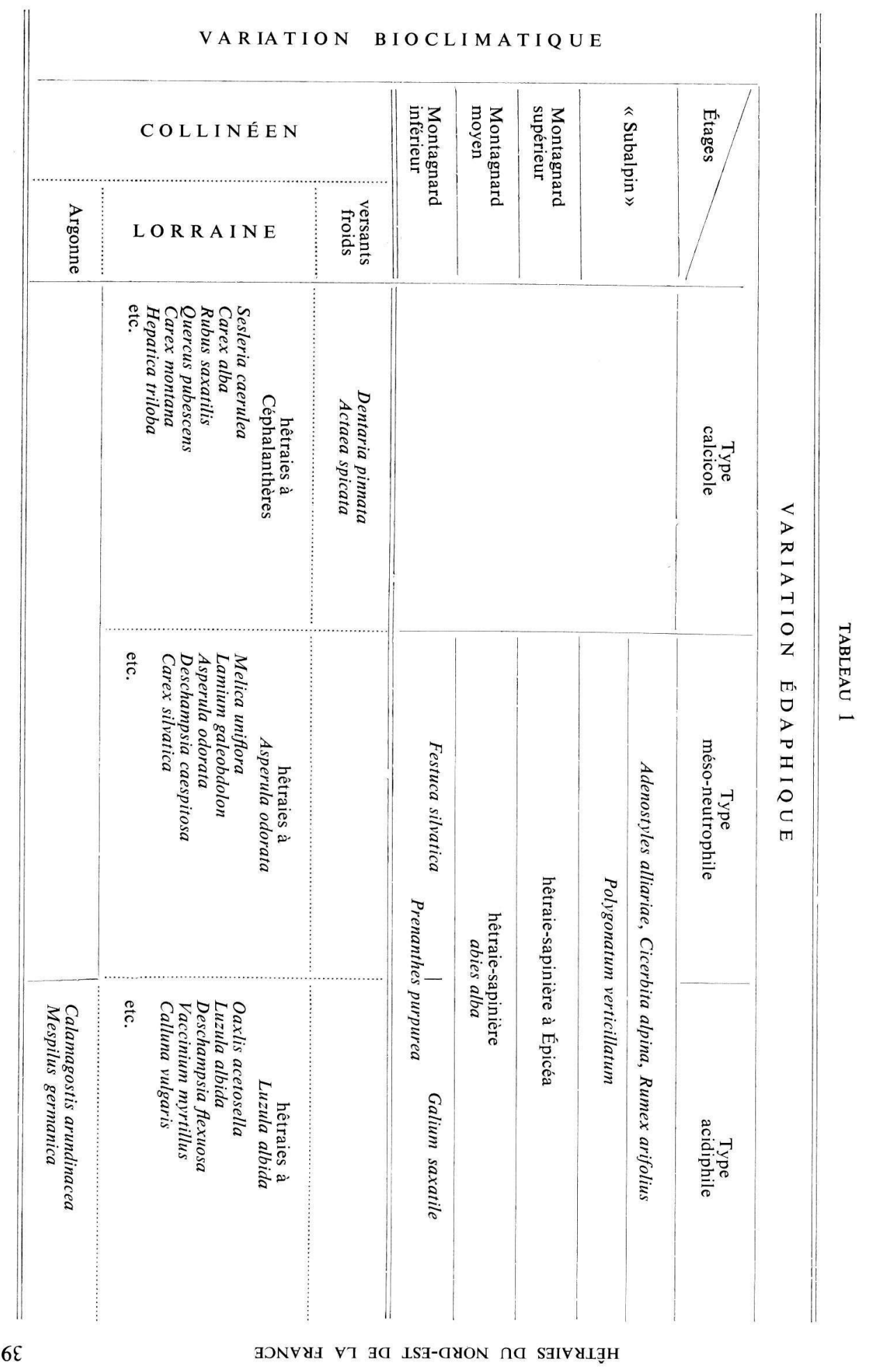




\title{
SUMMARY
}

\author{
MAIN ECOLOGICAL AND FLORISTIC \\ CHARACTERISTICS OF THE BEECH STANDS \\ IN THE NORTH-EAST OF FRANCE
}

This note gives a large view about the different kinds of Beech stands, which can be found at the various vegetation stages in the north-east of France. For each type informations are given about the determining ecological factors and about the species which characterize them best on a phytosociological point of view. A synthesis board tries to sum up all these types of Beech stands and to localize each of them, for one another, but also in relation to the ecological factors soils and climate

\section{RÉFÉRENCES BIBLIOGRAPHIQUES}

Becker M., 1972. - Étude des relations sol-végétation en condition d'hydromorphie dans une forêt de la plaine lorraine. Ann. Sci. forest., 1972, 30 (2), 143-182.

Bournerias M., 1968. - Guide des groupements végétaux de la région parisienne. Sedes — Paris, 1968.

CARBIEner R., 1964. - La détermination de la limite naturelle de la forêt par des critères pédologiques et géomorphologiques dans les Hautes-Vosges et le Massif-Central. C.R. Acad., Sci., 20/04/1964., E. 258, 4136-4138.

Carbiener R., 1966. - La végétation des Hautes-Vosges dans ses rapports avec les climats locaux, les sols et la géomorphologie, comparaison avec la végétation subalpine des montagnes d'Europe occidentale et centrale. Thèse Fac. Sci., Paris, 1966.

Chouard P., 1926. - Monographies phytosociologiques. II. La végétation des environs de Tonnerre (89) et des pays jurassiques au Sud-Est du Bassin de Paris. Bull. Soc. Bot. Fr., 73, 1006-1015, 1926.

Desplantes G., 1955/1958. - Additions et observations relatives à la Flore de Bourgogne et des régions limitrophes. Le Monde des plantes, 316, p. 12 et 325, p. 7.

Duchaufour Ph: 1957. - Tableau descriptif et analytique des sols. E.N.E.F., Nancy, 1957.

Duchaufour, Ph., 1960. - Stations, types d'humus et groupements écologiques. Rev. For. Fr., n 7, 1960 , 484-494.

Duchaufour Ph. et Bonneau M., 1960. - Les sols de la hêtraie en Europe occidentale. Bull. Inst. Agro. et Stat. Rech. Gembloux. (Série extraordinaire éditée à l'occasion du centenaire de l'Institut agronomique de 1'État), Vol. 1, 59-74.

Duchaufour Ph. et Jacamon M., 1958. - Les vallées des basses-Vosges et leurs types de forêt. Bull. Soc. Bot. Fr., 85 sess. extr. dans les Vosges et en Alsace, $106^{\mathrm{e}}$ année, Paris, 1958. For., t. 53, 262-270, 289-295, 321-325, 353-359.

Durin L. et Gehu J.-M., 1963. - Sur les hêtraies naturelles du N. O. de la France.

Duvigneaud J., 1961. - La végétation de l'entre-Sambre-et-Meuse : la hêtraie des sols calcaires. Bull. Soc. Roy. Bot. Belg., 93, 161-174.

Guinier Ph., 1955. - Les paysages végétaux des plateaux calcaires de l'Est du bassin parisien (forêts et friches). $81^{\text {e }}$ sess. extr. de la S.B.F. en 1953. Bull. Soc. Bot. Fr., 1955, 31-46.

IsSLER H., 1926. - Les associations végétales des Vosges méridionales et de la plaine rhénane avoisinante. Bull. Soc. Hist. Nat. Colmar, 1926, t. 7-20.

Issler E., 1931. - Les associations sylvatiques haut-rhinoises. Bull. Soc. Bot. Fr., t. 78, 1931

IssLer E., 1932. - Die Buchenwalde* der Hoch Vosegen (les forêts de Hêtre des Hautes Vosges), in «Die Buchenwalder Europas 》 de Rubel, 464-489.

JaCAMON M., 1959. - Le versant Ouest des Vosges in : $85^{\text {e }}$ sess. extr. de la S.B.F. Bull. Soc Bot. Fr., 1959, $1-10$.

Jacamon M. et Timbal J., 1972. - Projet de notice de la feuille de Nancy de la Carte de la végétation de la France au $1 / 200000^{\mathrm{e}}$. Document C.N.R.F. à diff. limit., 72/5/A.

Le Tacon F., et Timbal J., 1972. - A propos des conditions écologiques des hêtraies du Nord-Est et du Nord-Ouest de la France. Rev. For. Fr., t. 24, f. 3 1972, 187-200.

Le Tacon F. et Timbal J., 1973. - Valeurs indicatrices des principales espèces végétales des hêtraies du Nord-Est de la France vis-à-vis du type d'humus. Rev. For. Fr., t. 25, f. 4, 1973. 
Manil G., et al., 1963. - L'humus facteur de station dans les hêtraies acidiphiles de Belgique. Bull. Inst. Agr. et Stat. Rech. Gembloux, XXXI, 2, 1963, 183-222.

Mer E, 1917. - Associations de l'Épicéa au Sapin dans les sapinières des Hautes-Vosges. Rev. Eaux et For.

Moor M., 1952. - Die Fagion gessellschaften in Schweitzer Jura. 201 p. Berne, 1952.

Moor M., 1960. - Zur systematik des Querco-Fagetea. Mitt. der Floristich-soziologisch. Arbeits-gemersich. N. F., Heft. 8, 1960.

Moor M., 1972. - A. sociological and systematic classification of the Carici-Fagetum. Vegetatio. 1972, 24 (1/3), 31-69.

Noirfalise A., 1956. - La hêtraie ardenaise. Bull. Inst. Agr. et Stat. Rech. Gembloux, t. 30, fsc. 3-4, 1956.

Noirfalise A., 1962. - La hêtraie calcicole et ses taillis de substitution. Bull Inst. Agr. et Stat. Rech. Gembloux, t. XXX (3-4), 1962.

Noisette M., 1940. - La hêtraie des Basses-Vosges. Rev. Eaux et For., 1940, p. 1.

Oberdorfer, 1957. - Suddeutsche Pflanzengesell schaften.

PARDE J., 1962. - Sylviculture et production du Hêtre en Forêt de Haye (54) et en forêt de Retz (02). Stat. Rech. et Exp. For., Nancy, note techn. et forest. 15.

PiCard J.-F., 1970. - Les forêts sur rhétien dans le département des Vosges. Nouvelle contribution à la mise au point d'une méthode dynamique phyto-écologique du milieu forestier. Thèse $3^{\mathrm{e}}$ cycle, Nancy, 1968.

Rorsin P , 1967. - Contribution à l'étude du domaine phytogéographique atlantique et des hêtraies atlantiques d'Europe. Thèse, Gembloux, 1967.

RoL R., 1937. - Contribution à l'étude de la répartition du Sapin. (Abies alba Mill).

Rol R., 1954. - Végétation de la Lorraine et de l'Alsace. (Notices botaniques et itinéraire commenté publiés à l'occasion du $7 \mathrm{e}$ congrès international de Botanique, Paris, Nice, 1954.

Sougnez N., 1967. - Les forêts de la Lorraine belge. Duculot, édit., Gembloux, 1967.

Timbal J., 1968. - Contribution à l'étude des associations forestières de la forêt de Sainte-Hélène (88). Thèse $3^{\mathrm{e}}$ cycle, Fac. Sci. Orsay, 1968.

TUXen R., 1960. - Zur systematik der West-und-Mitteleuropaïschen Buchenwälder. Bull. Inst. Agr. et Stat. Rech. Gembloux (hors série), t. 1, 45-58.

VANDEN-Berghen C., 1956. - Remarque au sujet de la systématique des hêtraies de l'Europe occidentale. Bull. Soc. Roy. Bot. Belg., t. 89, p 15.

Vanden-Berghen C. et Couteaux M., 1955. - Contribution à l'étude de la végétation et de la Flore du District lorrain. III. Note sur la végétation forestière du district lorrain. Bull. Soc. Roy. Bot. Belg., 87, 2, 231-240.

Vanden-Berghen C. et Mullenders W., 1957. - Le Catena de Corniéville (Meuse, France). Bull. Soc. Roy. Bot. Belg., 90, 1, 63-71.

VAnden-Berghen C., et Mullenders W., 1957. - Étude sur les groupements végétaux des environs de Champlitte (plateau de Langres). Bull. Soc. Roy. Bot. Belg., 90, 1, 73-102.

Venet J., 1968. - La Lorraine. Fascicule de géographie forestière. E.N.G.R.E.F., Nancy, 1968. 


\section{ANNEXE \\ AMPLITUde ÉCOLOGIQUE D'UN CERTAIN NOMBRE D'ESPÈCES DES HÊTRAIES PAR RAPPORT AU TYPE D'HUMUS}

Ces résultats partiels sont tirés d'une étude plus générale effectuée en collaboration avec F. Le TACON de la station de pédologie du C. N. R. F. Ils ne sont valables au sens strict que pour l'étage collinéen.

\section{4. - tableau RÉCAPitulatif}

\section{Espèces principales des hêtraies}

1. Espèces acidiphiles

Calluna vulgaris

Leucobryum glaucum

Vaccinium myrtillus

Deschampsia flexuosa

Luzula albida

2. Espèces neutro-acidiphiles

Oxalis acetosella

Athyrium filix-femina

3. Espèces méso-neutrophiles

Milium effusum

Asperula odorata

Lamium galeobdolon

Carex silvatica

Melica uniflora

4. Espèces neutro-basophiles

Carex glauca

Carex digitata

Mercurialis perennis

5. Espèces thermo-xérophiles

Carex montana

Carex alba

Hepatica triloba

Sesleria caerulea

Différents types d'humus

Les numéros portés en abscisse correspondent aux divers types d'humus selon le code suivant :

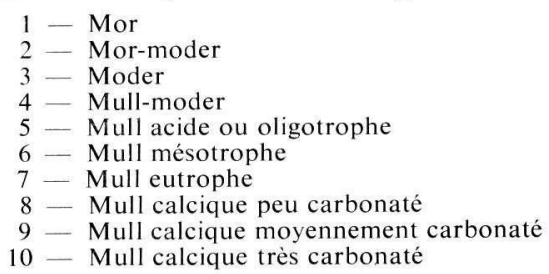

Cette échelle de 1 à 10 correspond, grosso-modo, à un gradient de $\mathrm{pH}$.

Remarque: Le trait plus gras indique une fréquence plus grande de l'espèce dans la classe d'humus correspondante, les pointillés à une interpolation. 
I. - EXEMPLE DE PROFILS D'ESPÈCES ACIDIPHILES

$\%$

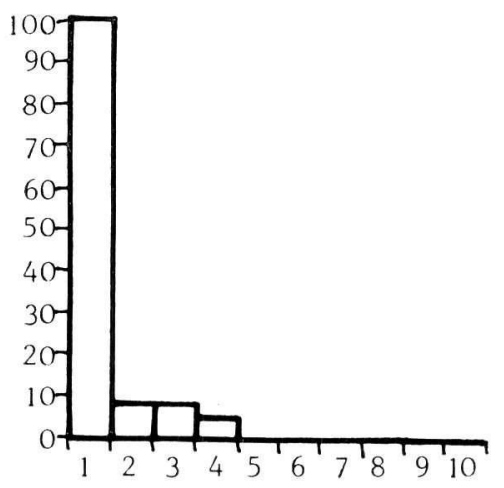

CALLUNA VULGARIS

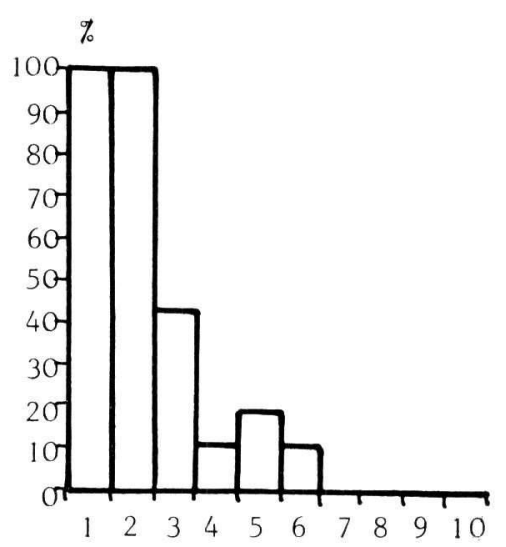

VACCINIUM MYRTILLUS

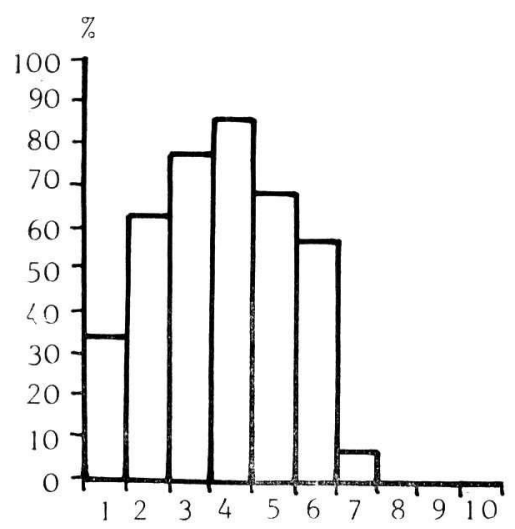

LUZULA ALBIDA
$\%$

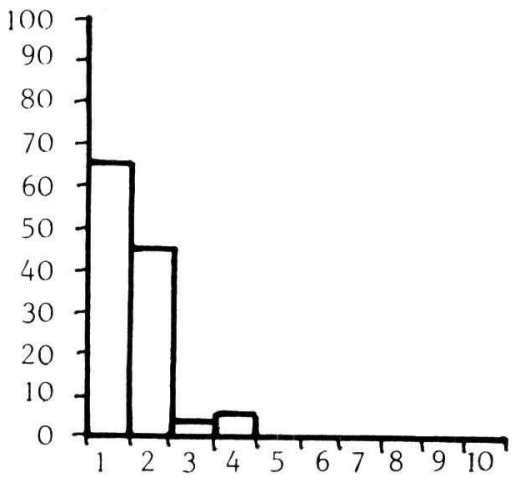

LEUCOBRYUM GLAUCUM

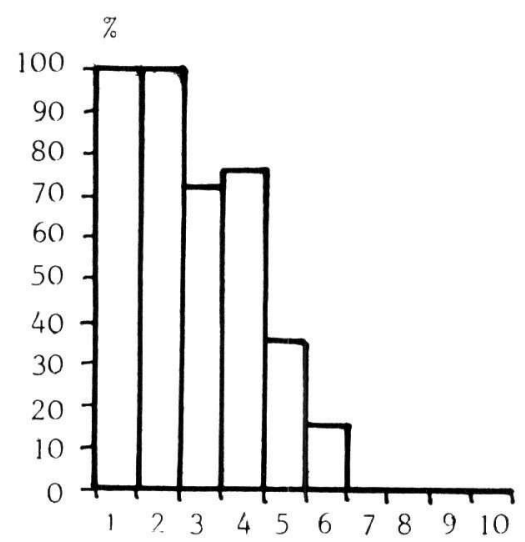

DESCHAMPSIA FLEXUOSA

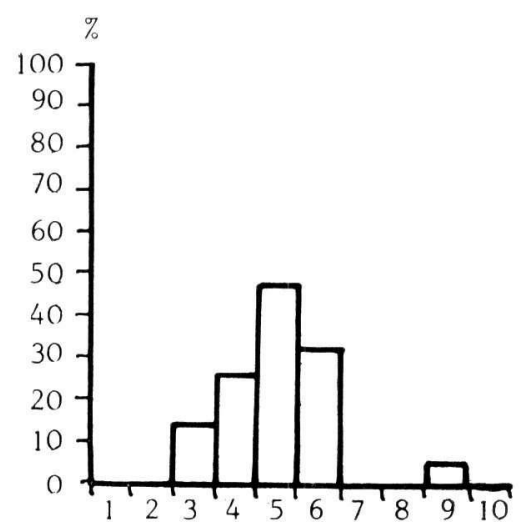


2. - EXEMPLE DE PROFILS D'ESPÈCES MESO-NEUTROPHILES
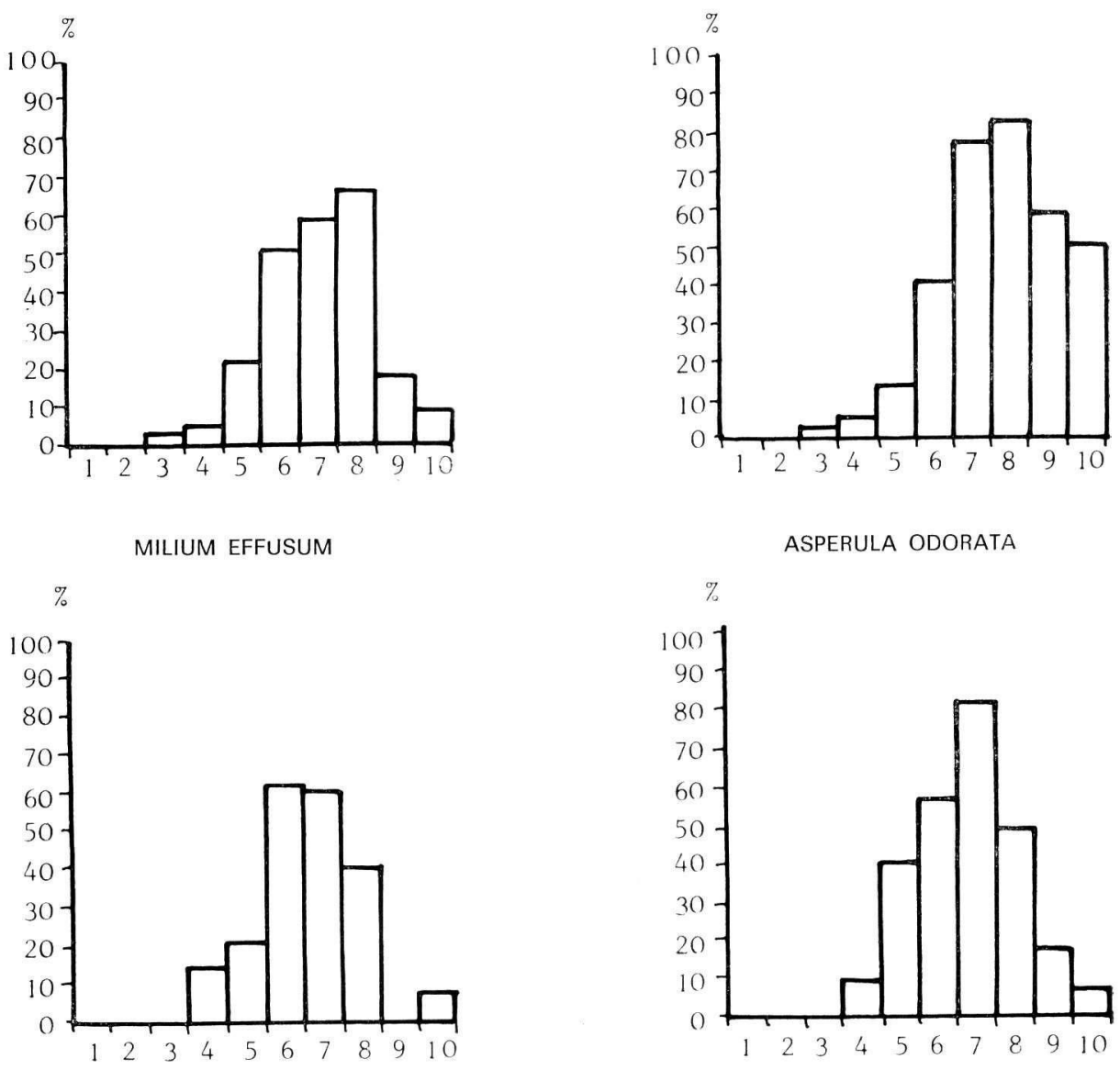

DESCHAMPSIA COESPITOSA

CAREX SILVATICA
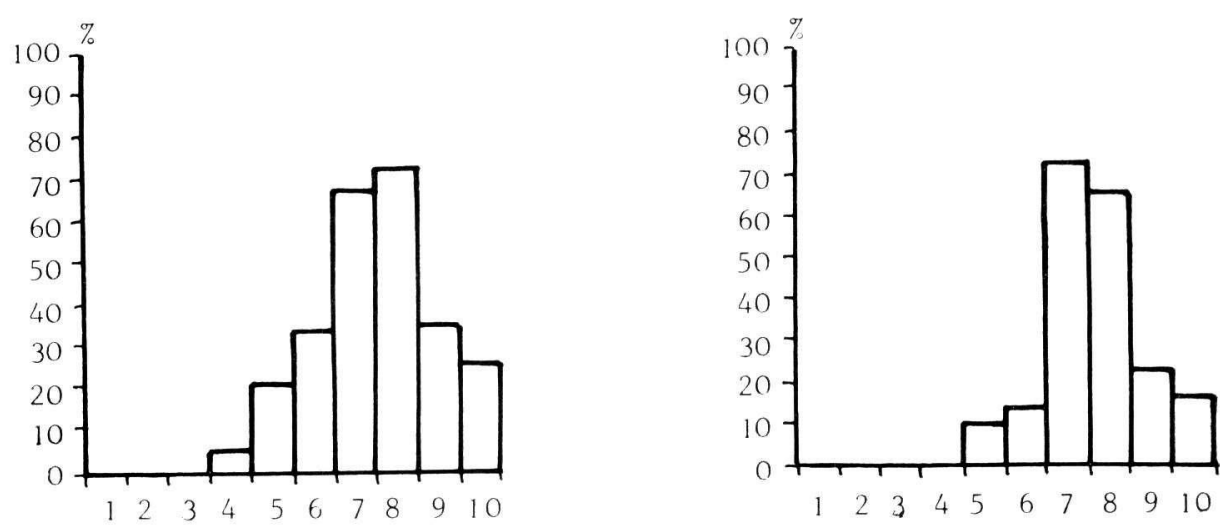

LAMIUM GALEOBDOLON 
3. - EXEMPLES DE PROFILS D'ESPÈCES « CALCICOLES》 ET THERMO-XÉROPHILES
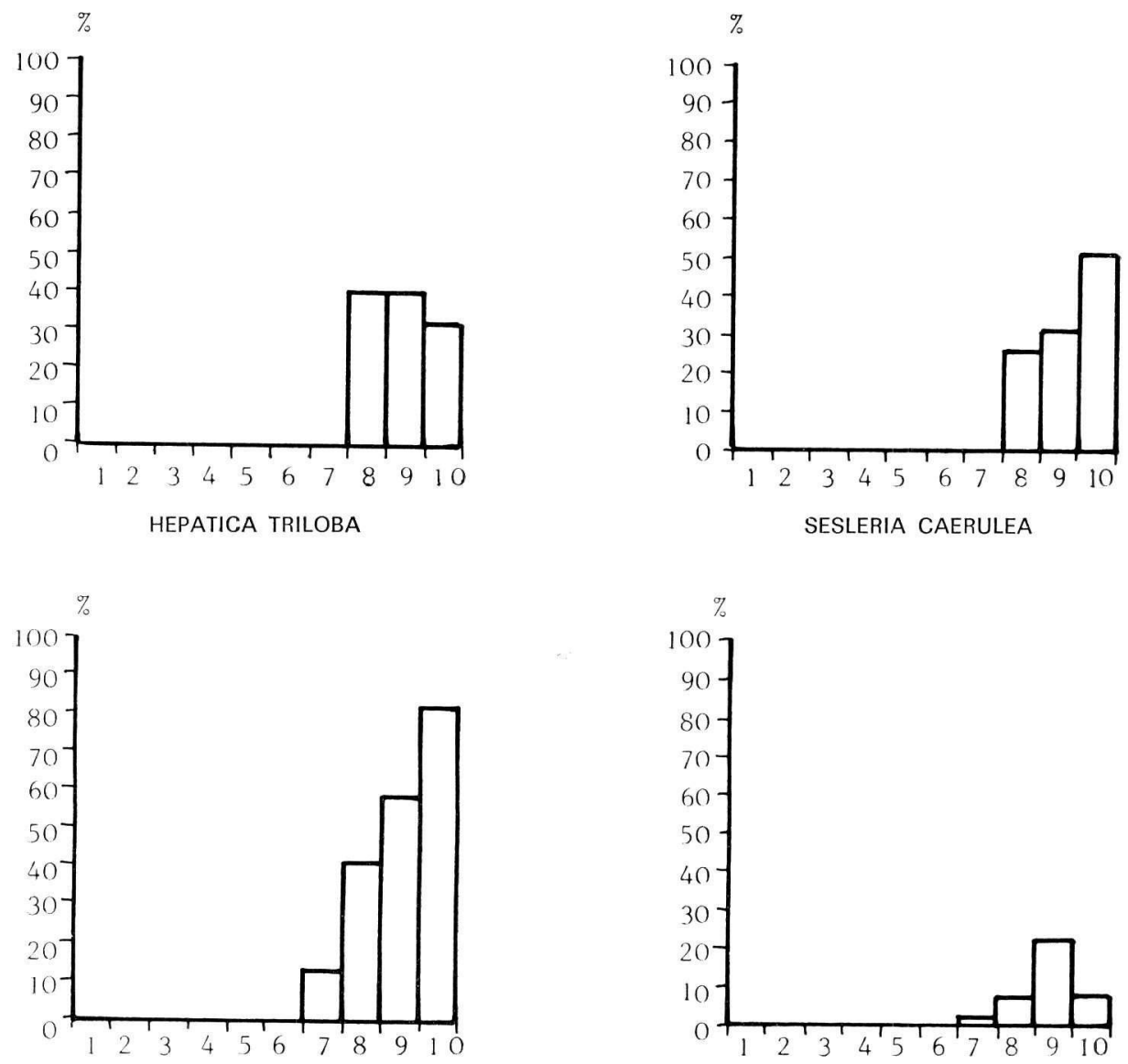

CAREX MONTANA CAREX ALBA

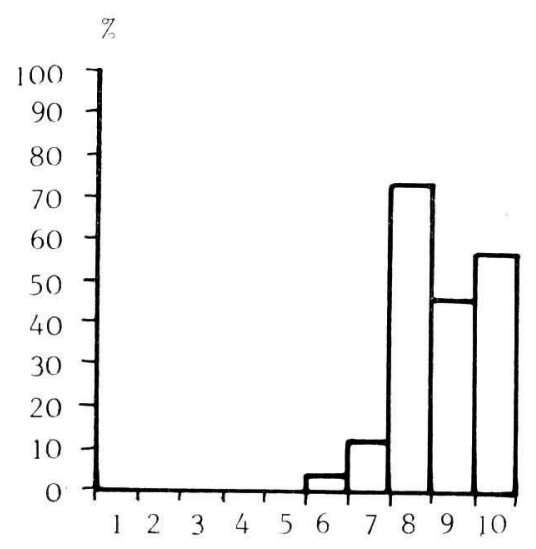

MERCURIALIS PERENNIS 